\title{
Prokaryotic ribosomal RNA stimulates zebrafish embryonic innate immune system
}

\author{
Abhishikta Basu, Maki Yoshihama, Tamayo Uechi and Naoya Kenmochi*
}

\begin{abstract}
Objectives: Cell-culture studies reported that prokaryotic RNA molecules among the various microbe-associated molecular patterns (MAMPs) were uniquely present in live bacteria and were categorized as viability-associated MAMPs. They also reported that specific nucleotide modifications are instrumental in the discrimination between self and nonself RNAs. The aim of this study was to characterize the in vivo immune induction potential of prokaryotic and eukaryotic ribosomal RNAs (rRNAs) using zebrafish embryos as novel whole animal model system. Additionally, we aimed to test the possible role of rRNA modifications in immune recognition.

Results: We used three immune markers to evaluate the induction potential of prokaryotic rRNA derived from Escherichia coli and eukaryotic rRNAs from chicken (nonself) and zebrafish (self). Lipopolysaccharide (LPS) of Pseudomonas aeruginosa served as a positive control. E. coli rRNA had an induction potential equivalent to that of LPS. The zebrafish innate immune system could discriminate between self and nonself rRNAs. Between the nonself rRNAs, $E$. coli rRNA was more immunogenic than chicken rRNA. The in vitro transcript of zebrafish $18 \mathrm{~S}$ rRNA gene without the nucleotide modifications was not recognized by its own immune system. Our data suggested that prokaryotic rRNA is immunostimulatory in vivo and could be useful as an adjuvant.
\end{abstract}

Keywords: rRNA, Lipopolysaccharide, MAMP, Zebrafish, Immunogenicity

\section{Introduction}

The recognition of microbe-associated molecular patterns (MAMPs) evokes the host innate immune system to induce downstream signaling pathways to eliminate the microbe. Among MAMPs, prokaryotic RNA molecules are distinctly called viability-associated MAMPs (vitaMAMPs) because of their unique association with live microbes. Since the innate immune system can discriminate live microorganisms from dead ones via recognition of vita-MAMPs, studies on immunostimulation potential of vita-MAMPs have attracted attention in the fields of vaccines and adjuvants [1]. The immune response induced by bacterial RNA upon recognition includes NF- $\kappa$ B-dependent proinflammatory cytokines, type I

*Correspondence: kenmochi@med.miyazaki-u.ac.jp

Frontier Science Research Center, University of Miyazaki, 5200 Kihara,

Kiyotake, Miyazaki 889-1692, Japan interferons and inflammasome activation [2]. In vitro studies suggested that RNA modifications could silence immune response and could contribute in the discrimination between self and nonself RNAs [3-5]. Eukaryotic ribosomal RNA (rRNA) possesses extensive modifications than prokaryotic rRNA. For example, human rRNA has at least 10 times more pseudouridine $(\Psi)$ and 25 times more 2'-O-methylated nucleotides than bacterial rRNA [3]. However, the role of rRNA in immune recognition is still underexplored.

In this study, we introduced zebrafish embryos as whole animal model system to characterize the in vivo induction potential of self and nonself (prokaryotic and eukaryotic) rRNAs. In addition, we compared the induction potentials of the in vitro transcribed zebrafish $18 \mathrm{~S}$ (IVT-18S) rRNA (devoid of any modifications) and native self rRNA to test the possible role of rRNA modifications in immune recognition. Zebrafish embryo is an attractive model system due to its small size, rapid life cycle, ex 
utero development, optical transparency and many other advantages [6]. We compared the induction potentials of the different rRNAs in zebrafish embryos with that of lipopolysaccharide (LPS) of Pseudomonas aeruginosa. LPS is a potent classic MAMP that is associated with the bacterial cell wall of both live and dead microbes. Our data demonstrated that zebrafish embryonic innate immune system could discriminate between externally administered self and nonself rRNAs, allowing induction of immune response specifically against the nonself rRNAs. The nonself prokaryotic rRNA was more potent stimulant than the nonself eukaryotic rRNA. Furthermore, the IVT-18S rRNA was not recognized by its own immune system. Taken together, our work reproduced some of the in vitro results of vita-MAMPs in a novel in vivo model using rRNA as the stimulant and suggested the possible use for prokaryotic rRNA in vaccines as adjuvant.

\section{Main text \\ Methods \\ Purification of rRNA}

The rRNAs were derived from Escherichia coli (nonself prokaryotic), chicken (nonself eukaryotic) and zebrafish (self). Liver from freshly sacrificed chicken (Gallus gallus domesticus) was purchased from a local meat shop. Zebrafish (Danio rerio) embryos were obtained by breeding the adults of $A B$ line at the animal house facility of University of Miyazaki following the animal welfare regulations (Authorization number: 2018-531). Total RNA was extracted from E. coli HST08 competent cells (TAKARA) (cultured for $2.5 \mathrm{~h}$ ) using the hot-phenol method and that from chicken liver and zebrafish embryos using TRI-reagent (Molecular Research Center Inc., USA). NucleoBond RNA/DNA 400 kit (MachereyNagel, Germany) was used to purify rRNA from total RNA. Following purification, rRNA was pelleted by isopropanol precipitation. The pellet was resuspended in water and quantified by NanoDrop measurement. Purity was confirmed by ratios of 260/280 and 260/230>1.9. The quality of the rRNA was assessed in MOPS denaturing gel (Additional file 1: Fig. S1).

\section{In vitro transcription}

Genomic DNA was extracted by heating one zebrafish embryo in $50 \mathrm{mM} \mathrm{NaOH}$ at $95^{\circ} \mathrm{C}$ for $10 \mathrm{~min}$. LA Takara kit and T7 promoter containing specific primers were used to amplify 18S rRNA region (Additional file 3: Table S1). Approximately 500 ng of the PCR product was in vitro transcribed using the T7-Scribe Standard RNA IVT kit (Cellscript, USA).

\section{Zebrafish embryos and microinjection}

Adult breeders of $A B$ lines were reared following the standard guidelines [7]. Zebrafish embryos at $30 \mathrm{~h}$ post fertilization (hpf) were anaesthetized in E3 medium containing $0.3 \mathrm{mg} / \mathrm{ml}$ tricaine methanesulfonate (Research Organics, USA) and then mounted onto presolidified $1 \%$ agarose gel-bed for microinjection. Test stimulants of $\sim 5.7 \mathrm{ng} / \mathrm{nl}$ concentration were injected into the yolk sac of embryos using IM-30 micromanipulator (Narishige, Japan). For the dose of $40 \mathrm{ng}, \sim 7 \mathrm{nl}$ volume was injected. Injection volume was measured by the standard micrometry method. Our experimental design is schematically represented in Additional file 2: Fig. S2. We used the induction levels of water-injected embryos as a control to quantitate the induction levels of test stimulants at the respective time points. Control embryos were injected with $\sim 7 \mathrm{nl}$ of water. Injected embryos were maintained in sterile E3 medium at $28.5^{\circ} \mathrm{C}$ until the incubation period. A batch of injected embryos was kept for observation until 3 days post injection ( $3 \mathrm{dpi}$ ).

\section{RNA quantitation}

Total RNA was extracted from a pool of eight embryos from three biological replicates. Approximately $1 \mu \mathrm{g}$ DNase-treated total RNA was reverse transcribed into cDNA using High-capacity cDNA Reverse Transcription kit (Applied Biosystems, USA). PCR was performed with a TAKARA thermocycler using Prime Taq DNA polymerase (GeNet Bio, Korea) and gene-specific primers for 35 cycles. Additional file 3: Table S1 provides the primer details of interleukin 8 gene (il8), pro-interleukin $1 \beta$ gene $(i l 1 \beta)$, proteasome subunit $\beta 9$ a gene (psmb9a) and ribosomal protein L38 gene (rpl38) used in this study. PCR products were run in $1.5 \%$ agarose gel electrophoresis using TAE buffer. Real-time or quantitative PCR (qPCR) was performed using Power SYBR Green PCR Master Mix (Applied Biosystems, USA) and the same primers in StepOnePlus Real-time PCR system (Applied Biosystems, USA). Changes in the transcript levels were normalized to that of rpl38 and expressed as fold changes relative to control using the $\Delta \Delta \mathrm{Ct}$ method. Student's t-test was applied to calculate $p$-value, and a value of $<0.05$ was considered statistically significant.

\section{Results \\ Bacterial rRNA shows immunogenicity similar to LPS}

Embryos were injected at $30 \mathrm{hpf}$ with various stimulants, and their induction potentials were compared at 2, 6 and 24 hpi by measuring the induced expression levels of $i l 8$, $i l 1 \beta$ and $p s m b 9 a$ genes. The markers IL8 and IL1 $\beta$ represent proinflammatory cytokines. Briefly, IL8 is a potent chemokine, and IL1 $\beta$ drives the expression of genes 


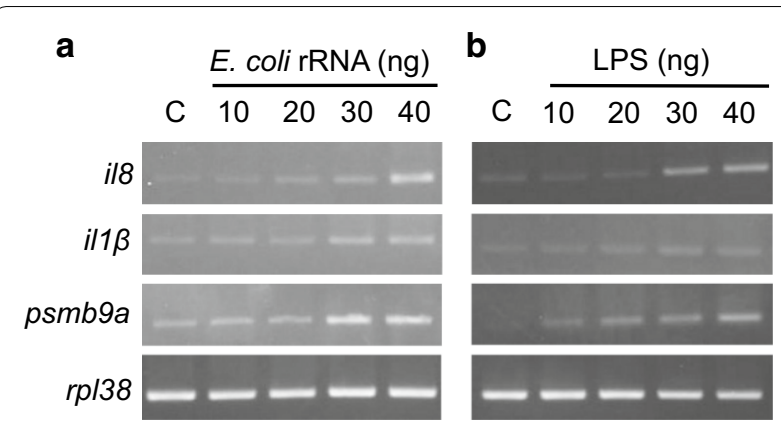

Fig. 1 Dose-dependent increase in the expression levels of markers upon E. coli rRNA and LPS stimulations. E. coli rRNA (10-40 ng) a and LPS (10-40 ng) b were injected into zebrafish embryos at $30 \mathrm{hpf}$. At $6 \mathrm{hpi}$, the induced expression levels of il8, il/ $\beta, p s m b 9 a$ and $r p / 38$ were analyzed by PCR for the respective doses of stimulants. C Control (water)

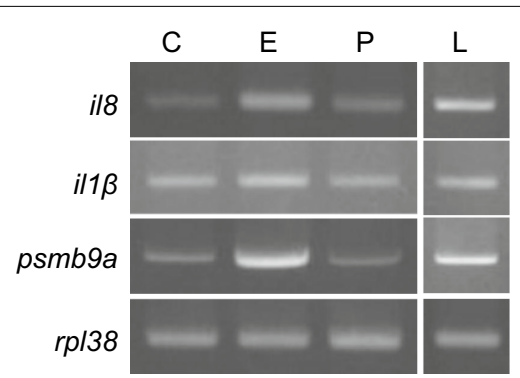

Fig. 2 Comparative induction potential of E. coli rRNA, poly (l:C) and LPS. Upon microinjection with $40 \mathrm{ng}$ of stimulants, the induced expression levels of $i / 8, i / 1 \beta$, psmb9a and rp/38 were analyzed at 6 hpi by PCR. C Control (water), EE. coli rRNA, P Poly (I:C) and L LPS

required for immune-mediated inflammation, effective adaptive immunity and antiviral control [8]. The other marker PSMB9A (also known as low molecular mass polypeptide 2 or LMP2) is a downstream target of interferon $\gamma$ [9] and is involved in MHC class I antigen presentation.

To determine a suitable stimulation dose, expression levels of immune markers at different amounts of $E$. coli rRNA and LPS were tested. At 40-ng dose, both E. coli rRNA and LPS stimulations induced enhanced expression of immune markers compared with control (Fig. 1). Additionally, the injected embryos of both the stimulants at this dose survived the observation period of $3 \mathrm{dpi}$ without any abnormalities (data not shown). Therefore, $40 \mathrm{ng}$ was standardized as the stimulation dose in this study.

When the induction potential of E. coli rRNA was compared with that of poly (I:C) and LPS, E. coli rRNA upregulated transcription of $i l 8$ and $p s m b 9 a$ similar to that of LPS (Fig. 2). Induced levels of $i l 1 \beta$ were inconclusive. In contrast, the expression levels of all the markers induced by poly (I:C) were similar to that of control. This result demonstrated that the immune response induced upon E. coli rRNA stimulation at the given conditions was similar to that of LPS and robust compared with that of poly (I:C). Therefore, we excluded poly (I:C) from further experiments and used only LPS as a positive control.

To validate our results, we quantitated the levels and studied the kinetics of induction upon $E$. coli rRNA and LPS stimulations in time-course experiments (Fig. 3a). E. coli rRNA and LPS displayed similar levels and kinetics of $i l 8$ and $p s m b 9 a$ induction, peaking at 2 and $6 \mathrm{hpi}$, respectively. In contrast, ill $\beta$ induction was highest at 2 hpi for LPS and 6 hpi for E. coli rRNA with same induction values. However, the induction levels at the respective time points were not significantly different between the two stimulants. Induction of all the markers by both the stimulants resolved by $24 \mathrm{hpi}$. Taken together, E. coli rRNA had equivalent immunogenicity as that of LPS in zebrafish embryos.

\section{Zebrafish embryos can discriminate between self and nonself rRNAs}

Next, we compared the induction levels and kinetics of zebrafish (self), chicken (nonself eukaryotic) and E. coli (nonself prokaryotic) rRNAs (Fig. 3b). Self rRNA did not induce any of the markers within the experimental time frame, implying that it skipped the immune recognition as expected. Chicken rRNA induced $i l 8$ and $i l 1 \beta$ expressions only at 2 hpi followed by resolution approximately $6 \mathrm{hpi}$. It did not induce psmb9a expression. Comparing the immunogenicity of the two nonself rRNAs, $E$. coli rRNA induced three markers for $24 \mathrm{~h}$, whereas chicken rRNA induced only two for approximately $6 \mathrm{~h}$. At $2 \mathrm{hpi}$, the induced expression level of $i l 8$ was higher for $E$. coli rRNA than chicken rRNA, but the values were not significantly different. On the other hand, the expression levels of $i l 1 \beta$ induction at $2 \mathrm{hpi}$ were same for both the nonself rRNAs (Fig. 3b). Taken together, the zebrafish embryonic innate immune system could discriminate between self and nonself rRNAs, and the immunogenicity of nonself prokaryotic rRNA was robust compared with nonself eukaryotic rRNA.

\section{In vitro transcribed self $18 \mathrm{~S}$ rRNA did not stimulate zebrafish embryos}

Previous studies with oligoribonucleotides and transfer RNA (tRNA) reported that the presence of 2'-O-methylated nucleotides and pseudouridine $(\psi)$ could silence immune response [3, 4]. To test whether nucleotide modifications of rRNA were involved in the discrimination of self rRNA, we generated the IVT-18S rRNA devoid of any modifications. Since 2 and 6 hpi were more critical in previous kinetics studies, we compared the induction potentials of IVT-18S rRNA and native self rRNA at these time points (Fig. 3c). Unexpectedly, IVT-18S rRNA 


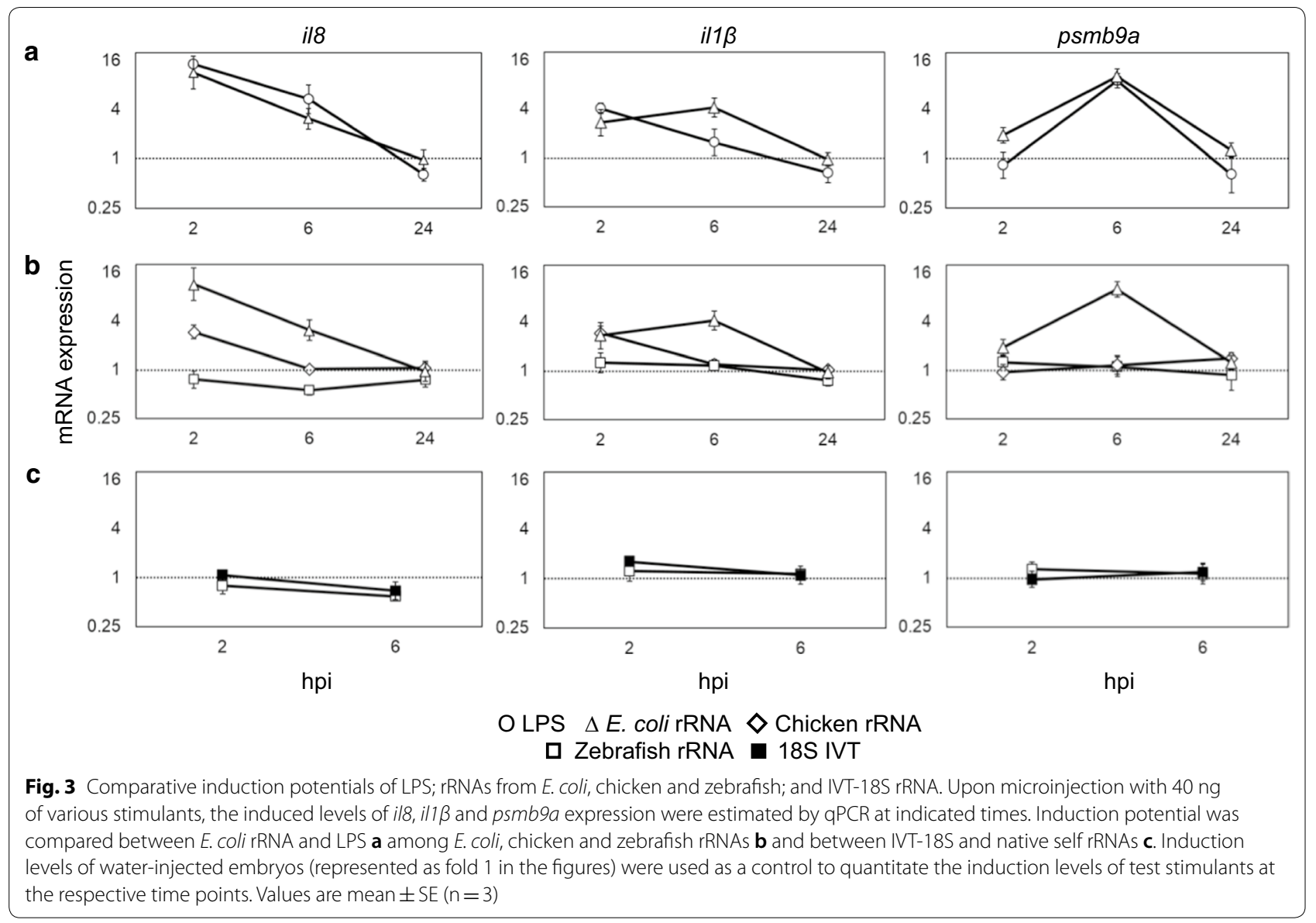

did not show any induction, as was observed in the case of native self rRNA. This result suggested that under the given conditions, the zebrafish innate immune system could not recognize either modified or unmodified self $18 \mathrm{~S}$ rRNA as an immunostimulant.

\section{Discussion}

In this study, we found that induction of ils upon stimulation was rapid, which correlated with the rapid il8 induction during acute inflammation reported in zebrafish larvae [10]. The peak induction of $p s m b 9 a$ at 6 hpi upon LPS stimulation was demonstrated previously [11]. Since the molecules involved in antigen processing are transcriptionally induced, even though adaptive immunity was not developed in zebrafish embryos [12], the same level of psmb9a induction at 6 hpi by both E. coli rRNA and LPS indicated their corresponding ability to induce adaptive immunity. The kinetics of $i l 1 \beta$ induction by live $E$. coli infection [13] precisely correlated with our data, thereby underscoring the immunogenicity of rRNA in zebrafish embryos.

The LPS-induced kinetics of $i l 1 \beta$ expression in our results were different from that of heat-killed E. coli stimulation [13], suggesting that the stimulation potential of individual MAMPs could be different from that of a combination of MAMPs presumably present in the heatkilled $E$. coli suspension. IL1 $\beta$ secretion is associated with bacterial viability [1]. Whether the prolong transcription of $i l 1 \beta$ induced by prokaryotic rRNA (vita-MAMP) compared with LPS (classic MAMP) as indicated by their different temporal peaks is a signature for viability sensing needs to be determined.

The robust immunogenicity of E. coli rRNA compared with chicken rRNA was corroborated by their respective antiviral activity [14]. Similar contrast in the induction potential of prokaryotic and eukaryotic RNA was also demonstrated between E. coli and mammalian total RNA [3] and tRNA [4]. Together, they confirmed that nonself prokaryotic rRNA was more potent than nonself eukaryotic rRNA. While the result of no induction upon stimulation by self rRNA was expected, that for IVT-18S rRNA was not. Although further investigation is required to confirm the role of nucleotide modifications, our findings suggested that under the given experimental conditions, nucleotide modifications of $18 \mathrm{~S}$ rRNA were not involved in the discrimination of self rRNA. Immune discrimination between self and nonself nucleic acids is not 
restricted to nucleotide modifications [4]. The immune system might discriminate self $18 \mathrm{~S}$ rRNA from the nonself by any particular conformation or sequence. Since the modifications within a specific sequence of E. coli $23 \mathrm{~S}$ rRNA was reported to abolish immune induction [2], the possible involvement of modifications in zebrafish $28 \mathrm{~S}$ rRNA needs to be investigated.

Monophosphoryl lipid A is a nontoxic derivative of LPS used as a licensed adjuvant for human use [1]. The overall similarities between the immunogenicity of $E$. coli $\mathrm{rRNA}$ and LPS in the present study together with the reported antiviral activity of $E$. coli rRNA [14] indicated that prokaryotic rRNA could have LPS-like adjuvant potential. The use of nonvirulent prokaryotic rRNA could be advantageous in augmenting the immunogenicity of live attenuated vaccines without the associated safety risks. Therefore, further examination determining the efficacy of prokaryotic rRNA as an adjuvant is worthy of consideration.

\section{Limitations}

In this study, we tested the induction potential of prokaryotic rRNA derived from only one source. Inclusion of other bacterial sources like Gram positive bacteria will widen the survey. We used three genes as the representative markers to evaluate the immune potential. Combination with the whole transcriptome analysis will improve our understanding of global immune induction. We tested induction only at transcription level. Testing the induction at translation level will substantiate our data. We used the unmodified in vitro transcribed $18 \mathrm{~S}$ zebrafish rRNA to investigate the role of nucleotide modifications in immunostimulation. This synthetic RNA partially represents the ideal unmodified version of native rRNA.

\section{Supplementary information}

Supplementary information accompanies this paper at https://doi. org/10.1186/s13104-019-4878-8.

Additional file 1: Fig. S1. MOPS gel shows the qualities of stimulant ribosomal rRNAs. Before microinjection, 100 ng of column purified rRNAs from E. coli, zebrafish and chicken were run in $1.5 \%$ MOPS denaturing agarose gel to assess their qualities.

Additional file 2: Fig. S2. The experimental design is represented schematically. Zebrafish embryos of AB line were treated with $0.003 \%$ bleach (Kao, Japan) to remove germs and raised in sterile $\mathrm{E} 3$ medium at $28.5^{\circ} \mathrm{C}$. At $24 \mathrm{hpf}$, the chorion layer was torn off (dechorionated). Embryos were injected at $30 \mathrm{hpf}$ with various samples, including LPS of Pseudomonas aeruginosa (Sigma, USA), poly (I:C) (Sigma, USA), water (control) and rRNAs of E. coli, chicken and zebrafish. Total RNA was extracted from a pool of 8 injected embryos at indicated time points to assess the induction level upon stimulation by qPCR. RT: Reverse transcription.

Additional file 3: Table S1. Primer sequences of immune markers and in vitro transcribed RNA.
Abbreviations

MAMPs: microbe-associated molecular patterns; vita-MAMPs: viability-associated MAMPs; hpf: hours post fertilization; hpi: hours post injection; rRNA: ribosomal RNA; tRNA: transfer RNA; LPS: lipopolysaccharide.

\section{Acknowledgements}

We thank Dr. Jun-ichi Hikima (Department of Biochemistry and Applied Biosciences, University of Miyazaki) and Mrs. Yukari Nakajima for their respective encouragement and technical support in the present study.

\section{Authors' contributions}

$A B$ and NK conceived and wrote the manuscript. $A B$ also performed the experiments and analysed the data. MY and TU advised on the study design, data analysis and revised the draft manuscript. All authors read and approved the final manuscript.

\section{Funding}

Not applicable.

\section{Availability of data and materials}

All data generated or analysed during this study are included in this published article and its supplementary information files. There isn't any sequencing data for sharing at the moment as no datasets have been generated or analyzed.

\section{Ethics approval and consent to participate}

All zebrafish experiments were performed according to the animal welfare regulations of University of Miyazaki (Authorization number: 2018-531).

\section{Consent for publication}

Not applicable.

\section{Competing interests}

The authors declare that they have no competing interests.

Received: 11 May 2019 Accepted: 26 December 2019

Published online: 03 January 2020

\section{References}

1. Blander JM, Barbet G. Exploiting vita-PAMPs in vaccines. Curr Opin Pharmacol. 2018;41:128-36.

2. Eigenbrod T, Dalpke AH. Bacterial RNA: an underestimated stimulus for innate immune responses. J Immunol. 2015;195:411-8.

3. Karikó K, Buckstein M, Ni H, Weissman D. Suppression of RNA recognition by toll-like receptors: the impact of nucleoside modification and the evolutionary origin of RNA. Immunity. 2005;2005(23):165-75.

4. Gehrig S, Eberle ME, Botschen F, Rimbach K, Eberle F, Eigenbrod T, Kaiser S, Holmes WM, Erdmann VA, Sprinzl M, Bec G, Keith G, Dalpke AH, Mark Helm M. Identification of modifications in microbial, native tRNA that suppress immunostimulatory activity. J Exp Med. 2012;209:225-33.

5. Keller P, Freund I, Marchand V, Bec G, Huang R, Motorin Y, Eigenbrod T, Dalpke A, Helm M. Double methylation of tRNA-U54 to 2'-O-methylthymidine (Tm) synergistically decreases immune response by Toll-like receptor 7. Nucleic Acids Res. 2018;46:9764-75.

6. Varela M, Figueras A, Novoa B. Modelling viral infections using zebrafish: innate immune response and antiviral research. Antiviral Res. 2017;139:59-68.

7. Westerfield M. The Zebrafish book: a guide for the laboratory use of zebrafish (Danio rerio). 4th ed. Eugene: University of Oregon Press; 2000

8. Aarreberg LD, Wilkins C, Ramos HJ, Green R, Davis MA, Chow K, Gale M Jr. Interleukin-1 $\beta$ signaling in dendritic cells induces antiviral interferon responses. MBio. 2018;9:e00342-18.

9. Sieger D, Stein C, Neifer D, Van der Sar AM, Leptin M. The role of gamma interferon in innate immunity in the zebrafish embryo. Dis Model Mech. 2009:2:571-81.

10. De Oliveira S, Reyes-Aldasoro CC, Candel S, Renshaw SA, Mulero V, Calado A. CxCl8 (IL-8) mediates neutrophil recruitment and behavior in the zebrafish inflammatory response. J Immunol. 2013;190:4349-59.

11. Marqués L, Brucet M, Lloberas J, Celada A. STAT1 regulates lipopolysaccharide- and TNF-a-dependent expression of transporter associated with 
antigen processing 1 and low molecular mass polypeptide 2 genes in macrophages by distinct mechanisms. J Immunol. 2004;173:1103-10.

12. Stockhammer OW, Zakrzewska A, Hegedûs Z, Spaink HP, Meijer AH. Transcriptome profiling and functional analyses of the zebrafish embryonic innate immune response to Salmonella infection. J Immunol. 2009;182:5641-53.

13. Sander LE, Davis MJ, Boekschoten MV, Amsen D, Dascher CC, Ryffel B, Swanson JA, Müller M, Blander JM. Detection of prokaryotic mRNA signifies microbial viability and promotes immunity. Nature. 2011;474:385-9.
14. Stewart AG, Grantham CA, Dawson KM, Stebbing N. The antiviral activity of ribosomal polynucleotides against encephalomyocarditis virus infection of mice. Arch Virol. 1980;66:283-91.

\section{Publisher's Note}

Springer Nature remains neutral with regard to jurisdictional claims in published maps and institutional affiliations.
Ready to submit your research? Choose BMC and benefit from:

- fast, convenient online submission

- thorough peer review by experienced researchers in your field

- rapid publication on acceptance

- support for research data, including large and complex data types

- gold Open Access which fosters wider collaboration and increased citations

- maximum visibility for your research: over $100 \mathrm{M}$ website views per year

At BMC, research is always in progress.

Learn more biomedcentral.com/submissions 